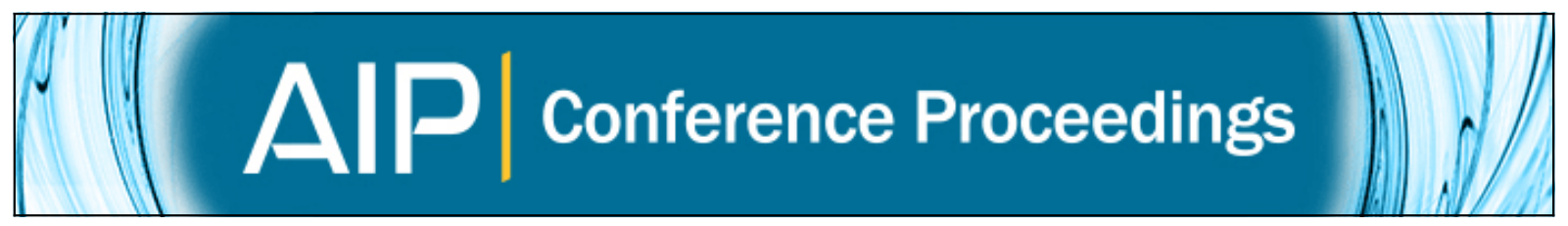

\title{
Preparation of nanofluids based on solar salt and boehmite nanoparticles: Characterization of starting materials
}

B. Muñoz-Sánchez, J. Nieto-Maestre, I. Iparraguirre-Torres, J. A. Sánchez-García, J. E. Julia, and A. GarcíaRomero

Citation: AIP Conference Proceedings 1734, 050030 (2016); doi: 10.1063/1.4949128

View online: http://dx.doi.org/10.1063/1.4949128

View Table of Contents: http://scitation.aip.org/content/aip/proceeding/aipcp/1734?ver=pdfcov

Published by the AIP Publishing

Articles you may be interested in

The influence of mixing water on the thermophysical properties of nanofluids based on solar salt and silica nanoparticles

AIP Conf. Proc. 1734, 050031 (2016); 10.1063/1.4949129

Preparation and characterization of ambazone salt with nicotinic acid

AIP Conf. Proc. 1425, 30 (2012); 10.1063/1.3681959

Preparation and Characterization of Calcium Carbonate Nanoparticles

AIP Conf. Proc. 1284, 195 (2010); 10.1063/1.3515549

Nanofluid-based direct absorption solar collector

J. Renewable Sustainable Energy 2, 033102 (2010); 10.1063/1.3429737

Preparation and characterization of Dy nanoparticles

Appl. Phys. Lett. 74, 1478 (1999); 10.1063/1.123586 


\title{
Preparation of Nanofluids Based on Solar Salt and Boehmite Nanoparticles: Characterization of Starting Materials
}

\author{
B. Muñoz-Sánchez ${ }^{1,3}$, J. Nieto-Maestre ${ }^{1, a)}$, I. Iparraguirre-Torres ${ }^{1}$, J.A. Sánchez- \\ García $^{1}$, J.E Julia ${ }^{2}$ and A. García-Romero ${ }^{3}$ \\ ${ }^{1}$ Tecnalia Research and Innovation, Mikeletegi Pasealekua, 2. 20009 - San Sebastián (Gipúzcoa). Spain. \\ ${ }^{2}$ Department of Mechanical Engineering and Construction, Universitat Jaume I. Campus de Riu Sec. 12071 - \\ Castellón de la Plana (Castellón). Spain. \\ ${ }^{3}$ Department of Mining Engineering, Metallurgy and Materials Science, University of the Basque Country \\ (UPV/EHU), Rafael Moreno "Pitxitxi”, 2. 48013 - Bilbao (Vizcaya). Spain.
}

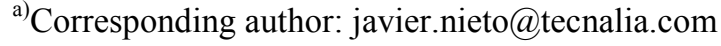

\begin{abstract}
A nanofluid composed of Solar Salt (SS) and boehmite nanoparticles (A) in a concentration of $1 \%$ by weight, is proposed as thermal storage medium for Concentrated Solar Power (CSP) plants. A wide characterization of the raw materials has been done, focused on their thermal stability and the nanoparticle primary size and shape among other properties such as its specific heat and crystalline structure. Some features of the final nanofluids have been also investigated: thermal stability, nanoparticle sizes and their distribution and specific heat. The showed results confirm that these materials are thermally stable in the working temperature range both individually and combined. In addition, the synthesis procedure implemented is effective to keep the nanoparticle sizes in the nanometric range $(<100 \mathrm{~nm})$. These findings mean the first step to carry on research and characterization of this nanofluid.
\end{abstract}

\section{INTRODUCTION}

Feasibility of solar thermal technology has been widely demonstrated, becoming a safe bet in the energy mix. Growing interest from both public and private sector has led to achieve considerable advances and improvements in recent years. However, one of the critical factors to make this energy option more competitive is to extend plant operation time when sun is not available (cloudy weather or at night). This would allow to produce energy in a constant way and to adapt operation to energy demand. In this way, there is a need of storing the thermal energy when sun is shining to use it afterwards.

The current system employed in CSP plants consists of an inorganic salt composed of an eutectic mixture of sodium and potassium nitrate (60:40 by weight) which store the energy as sensible heat. It is known as SS and the configuration is the two-tank molten salt system. This salt is cheap, environmentally friendly and thermally stable at the temperatures of the CSP installation, which makes it interesting as thermal storage medium. However, its main drawback is its poor heat transfer rate due to the low thermophysical properties, such as the specific heat $\left(\mathrm{c}_{\mathrm{p}}\right)$ and thermal conductivity $(\mathrm{k})$ in the liquid state. This implies the need of a huge quantity of material to store the required energy for the solar plant. In this context, effective solutions are needed to increase the heat transfer characteristics of this material.

Nanofluids have been recently proposed as a successful answer to the mentioned issue. Nanofluids are described as liquid suspensions containing nanometre-sized particles $(<100 \mathrm{~nm})$. They have been widely studied for the last twenty years as a way to increase the thermal conductivity of the base fluid. Inside this broad research topic, the specific study of molten-salt based nanofluids is quite recent (first works date from 2010 [1]) and this technology is still at its infancy. As a consequence, there are some controversial published results related to the $c_{p}$ enhancement of 
the inorganic salts when nanoparticles are present. The mechanism that is governing this behaviour is still not very well-known, though it seems clear that the available nanoparticle surface area plays an important role $[2,3]$.

In this work, a nanofluid based on SS containing boehmite nanoparticles in a concentration of $1 \%$ by weight is studied. In particular, we have developed a specific characterization of the initial materials as a starting point to create a nanofluid with improved thermal properties. Some other properties have been measured in the final material, mainly to assure that nanometric sizes are kept after the manufacturing procedure and the nanoparticles have not been highly agglomerated.

\section{EXPERIMENTAL}

\section{Raw Materials}

\section{Boehmite Nanoparticles}

The starting material was Alumisol 10A, an industrial water nanofluid (Kawaken Fine Chemicals, Co.) containing $10 \%$ by weight of boehmite nanoparticles with a primary size of $10 \mathrm{~nm}$ according to the manufacturer.

$$
\text { Solar Salt }
$$

Solid pellets of sodium nitrate (Industrial grade, SQM industries) and potassium nitrate (Multi-KGG Greenhouse-grade, Haifa Group) were crushed in a mortar and mixed in the proper quantities to obtain the desired composition $\left(60: 40\right.$ by weight of $\left.\mathrm{NaNO}_{3}: \mathrm{KNO}_{3}\right)$.

\section{Nanofluid Synthesis Procedure}

Boehmite nanoparticles were included in the SS at a concentration of $1 \%$ by weight to create the nanostructured material (SS1A). The synthesis was performed as follows. Initial materials were dissolved in water to obtain initial solutions of $15 \%$ and $1 \%$ by weight for SS and A respectively. The necessary amounts of these solutions were mixed under agitation. The water was removed by simple heating $\left(100^{\circ} \mathrm{C}\right)$ in a furnace and finally the material was melted at $450{ }^{\circ} \mathrm{C}$ in an oven for 30 minutes to homogenize the sample. After cooling it at room temperature, the solid material was milled again and a representative portion was obtained.

\section{Characterization Methods}

Thermogravimetric Analysis (TGA)

The thermal stability both of the starting materials (A and SS) and the final nanofluid (SS1A) was analysed. About $20 \mathrm{mg}$ of the dried samples were put in a platinum crucible and heated in a TGA device (SETARAM SETSYS Evolution - 1750, Setaram Instrumentation, France). The heating program consisted of a first heating from 20 to $90{ }^{\circ} \mathrm{C}$ at $5{ }^{\circ} \mathrm{C} / \mathrm{min}$ to remove humidity followed by a second stage from 90 to $550{ }^{\circ} \mathrm{C}$ at $2{ }^{\circ} \mathrm{C} / \mathrm{min}$. Then, the sample was maintained at the highest temperature for 3 hours. Finally, it was submitted to two thermal cycles of heating and cooling between 90 and $550{ }^{\circ} \mathrm{C}$ to know if possible changes are reversible or not. The analyses were always done under air atmosphere.

\section{X-Ray Diffraction (XRD)}

A XRD device Bruker D8Advance was used to have information about the crystal structure of the raw materials A and SS. For the boehmite, two different spectra were taken: after simple drying of Alumisol $\left(100^{\circ} \mathrm{C}\right)$ and after applying the same thermal treatment of the nanostructured material $\left(450{ }^{\circ} \mathrm{C}, 30 \mathrm{~min}\right)$ in order to know its final conformation. A comparison between the boehmite structure before and after this thermal program was done. In addition, SS spectrum was compared with other bibliographic sources. 
The nanoparticle size distribution of A and SS1A was characterized using the DLS technique (ZetaSizer nano ZS, Malvern Instruments, Ltd. Malvern, UK). The starting boehmite dispersion Alumisol 10A was used to measure the initial sizes and the final nanofluid SS1A was measured after melting, cooling it at room temperature and carefully milling. A certain quantity of distilled water is needed to be added to the sample prior to the analysis according to their nanoparticle expected size. The nanoparticle concentration was $0.5 \mathrm{mg}$ per $\mathrm{mL}$ of water for the starting boehmite and $0.1 \mathrm{mg} / \mathrm{mL}$ for the synthetized material, in agreement with Malvern experts' advices. The presence of dissolved nitrates in SS1A was taken into account into the operating procedure implemented on the device to analyse this sample.

\section{Transmission Electron Microscopy (TEM)}

The size and shape of primary boehmite nanoparticles were observed by means of TEM using a device JEOL 2100 operating at a voltage of $100 \mathrm{kV}$. The elemental composition of boehmite was analyzed by means of Energy Dispersive X-ray Spectroscopy using an EDX system (Oxford Instruments INCA Penta FETX3) attached to TEM.

\section{Scanning Electron Microscopy (SEM)}

The dispersion of nanoparticles in solid SS1A samples was evaluated using a field emission scanning electron microscope (SEM) (JEOL, 7001F). Specimens were metallized in a thermal evaporator. Secondary Electrons (SE) and Quadrant Back-Scattering Detector (QBSD) images and digital image processing were used to characterize the nanoparticle clusters in the solid salt. The elemental composition of the material was analyzed by means of Energy Dispersive X-ray Spectroscopy using an EDX system (Oxford Instruments INCA Penta FETX3) attached to SEM.

\section{Differential Scanning Calorimetry (DSC)}

A DSC device (Q100, TA Instruments, US) was used to measure the specific heat $\left(\mathrm{c}_{\mathrm{p}}\right)$ of the raw materials A, SS and SS1A through Modular Differential Scanning Calorimetry (MDSC) with an isothermal procedure. Three temperatures, $196^{\circ} \mathrm{C}$ (SS in the solid state), $256^{\circ} \mathrm{C}$ and $396^{\circ} \mathrm{C}$ (SS in the liquid state) were tested. Some milligrams of the sample were introduced in an aluminum pan hermetically sealed under an inert atmosphere. The samples were first stabilized at the desired temperature and then subjected to an isothermal period of 10 minutes to achieve the salt thermal equilibrium prior to the data collection ( 20 minutes). The modulation was set up with $0.6^{\circ} \mathrm{C}$ of amplitude and a 110 seconds period according to the TA Instruments expert advices. The explained isothermal program was always performed from the highest to the lowest temperature. Thus, in the case of SS a complete melting of the sample was achieved at the beginning of the experiment (melting temperature $220^{\circ} \mathrm{C}$ ). In this way, the sample inside the crucible in the solid state behaves as a compact piece rather than the initial fine powder. The sapphire sample commonly used as the reference material was first measured under the same protocol to find out the possible device divergence and correct the final sample results.

Three different replicas were measured for each sample and the average $c_{p}$ and its Standard Deviation (SD) were obtained.

\section{RESULTS AND DISCUSSION}

\section{Thermal Stability}

The first and most important step to validate the feasibility of a certain nanomaterial to be included in a matrix of molten salt is its thermal stability. It is essential to analyze both the possible changes of the nanomaterial and also those of the mixture with the salt at the working temperature range. Figure 1 shows the thermogravimetric (TG) curves from $20{ }^{\circ} \mathrm{C}$ to $550{ }^{\circ} \mathrm{C}$ in the first thermal cycle for A, SS and the nanostructured material SS1A. The differential thermogravimetric (dTG) curve is also presented in the case of A to analyze the inflections in the TG curve and consequently the changes in the rate of weight loss.

The thermal analysis of boehmite nanoparticles revealed three different stages in our temperature range. The first weight loss $(\Delta \mathrm{m}=-8.4 \%)$ occurs between 20 and $90^{\circ} \mathrm{C}$ with a minimum around $80^{\circ} \mathrm{C}$, due to the loss of humidity of 
the sample. A second step of dehydration $(\Delta \mathrm{m}=-13.3 \%)$ is observed in the range of $90-400{ }^{\circ} \mathrm{C}$ with an endothermic peak in $390{ }^{\circ} \mathrm{C}$ related to the desorption of intralaminar water located between boehmite nanoparticles. Finally, the boehmite starts the transformation in $\gamma-\mathrm{Al}_{2} \mathrm{O}_{3}$ that accounts the $5.6 \%$ of the mass loss. These results are in agreement with literature on this subject $[4,5]$.

According to the curves, the SS is thermally stable and the weight losses are minor $(0.6 \%)$. It is considered that formation of nitrites (the main mechanism of thermal degradation of nitrates) starts around $450{ }^{\circ} \mathrm{C}$ but at temperatures lower than $600{ }^{\circ} \mathrm{C}$ it occurs in a very little extension [6].

The curve which represents the SS1A sample is very similar to that of the SS and mass losses are slightly higher due to the presence of boehmite and its dehydration processes explained before.

The three samples registered neglectful weight losses (under the detection limit of the device) after their thermal cycling, so it is considered that any thermal events found in the first heating cycle are irreversible. This is of quite importance because a stable sample of solar salt with boehmite nanoparticles can be obtained by melting it at the suitable temperature. Therefore, it is considered that boehmite is a valid nanomaterial to be mixed with the solar salt.

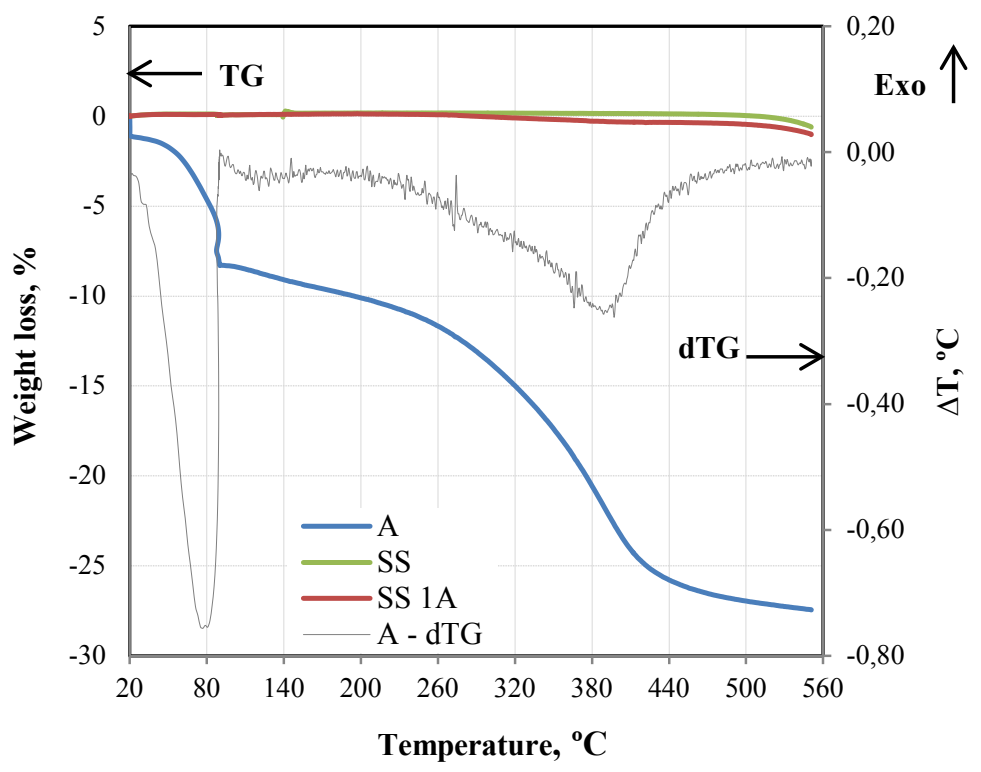

FIGURE 1. TG-dTG curves of the initial boehmite (A), Solar Salt (SS) and solar salt with boehmite nanoparticles (SS1A).

\section{Crystalline Structure}

Figure 2 exhibits the recorded XRD patterns for A (before and after calcination) and SS. The spectrum of boehmite nanoparticles showed several peaks typical of this material as is supported by XRD spectra database software of the device and the specialized literature [6]. After the thermal treatment at $450{ }^{\circ} \mathrm{C}$, there is a decrease of crystallinity and the pattern is more diffused. This is probably due to the loss of intralaminar water and the incipient conversion to $\gamma-\mathrm{Al}_{2} \mathrm{O}_{3}$, which was revealed by the TG tests results.

On the other hand, the peaks appearing in the SS spectra are also in agreement with the aforementioned database. This XRD pattern will be further employed in the nanostructured samples to get knowledge about possible changes happened in the crystalline structure of SS induced by the addition of boehmite. 


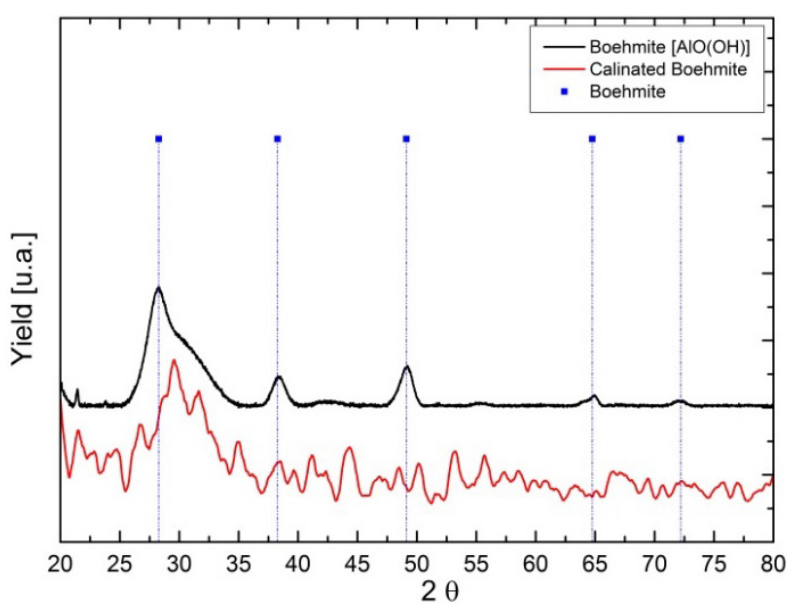

(a)

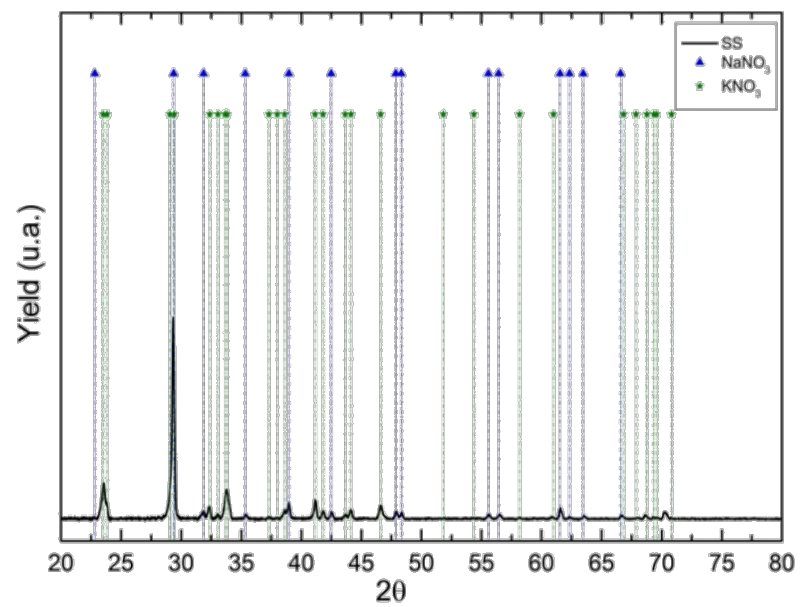

(b)

FIGURE 2. XRD spectra of boehmite before and after calcination process (a) and Solar Salt (SS) (b).

\section{Nanoparticle Size, Morphology and Distribution}

It is generally accepted that the nanoparticle size in the final synthetized material is the key factor responsible of the anomalous increment of the thermophysical properties in the molten-salt based nanofluids [2,3]. Therefore, it is of high importance to control and limit the nanoparticle agglomeration through the synthesis procedure in order to stay in the nanometric range $(<100 \mathrm{~nm})$. The nanoparticle shape may have also some influence on the further interaction with the anions and cations of the SS. The distribution of nanoparticles into the salt matrix is also interesting to discard a high heterogeneity of the sample.

The size distribution of A and SS1A obtained from DLS is shown in Fig. 3. The boehmite initial sizes are in the range from 8 to $25 \mathrm{~nm}$ with a maximum in $13 \mathrm{~nm}$. Bigger sizes are found in the sample SS1A due to the nanoparticle agglomeration occurred during the preparation of the nanostructured material. Most of the nanoparticles sizes falls in the interval 50 to $140 \mathrm{~nm}$ and the maximum is located in $68 \mathrm{~nm}$. A tiny quantity of the nanoparticles suffered a higher degree of agglomeration with a size around $712 \mathrm{~nm}$.

Figure 4 presents two TEM images of the initial boehmite. On the one side, the Fig. 4 (a) gives an idea of the nanoparticle size which is in the range of that obtained by DLS technique. On the other side, it can be deduced from both images that the morphology of the boehmite nanoparticles consists of several layers arranged in a fiber-like structure.

Finally, SEM images of SS1A were examined to get knowledge about the clusters sizes and its distribution along the SS matrix. Images taken on the solid sample both with the SE and QBSD detector can be seen in Fig. 5 (a) and (b) respectively, along with their elemental composition from EDX analysis. These images are similar to the ones published by other research groups working with nitrate salts [2,3]. The matrix of salt is seen as a group of globeshaped crystals with two different gray tones corresponding to the components of $\mathrm{SS}\left(\mathrm{NaNO}_{3}\right.$ in darker gray). This color differences can only be seen in QBSD images (Fig. 5 (b)). The nanoparticles appear embedded in the SS crystals with a different shape. The marked-red areas were analyzed with EDX to confirm the presence of nanoparticles. In Fig. 5 (a) an agglomerate of around $5 \times 3 \mu \mathrm{m}$ was found and its elemental composition revealed that aluminum is present in that area. Some quantity of chloride was also found because this element is contained in the industrial-grade nitrates which have been employed. The cluster analyzed in the indicated area in Fig. 5 (b) is about $1 \mu \mathrm{m}$ in size and aluminum appears in the elemental composition. Some carbon appears due to contamination of the sample and little quantities of chloride were found again. It is worth mentioning that in both Fig. 5 (a) and 5 (b) the EDX analyses were also performed in areas outside those marked in red. No aluminum was found in those regions.

The analyzed DLS results show that the manufacturing method followed to produce SS1A is adequate since most of the nanoparticles remains in the nanometric range in the final material. This is an important first step that would allow achieving an enhancement in $\mathrm{c}_{\mathrm{p}}$ when further DSC analyses were done. In addition, the microscope images have confirmed the size distributions of nanoparticles both before and after adding them in the SS. 


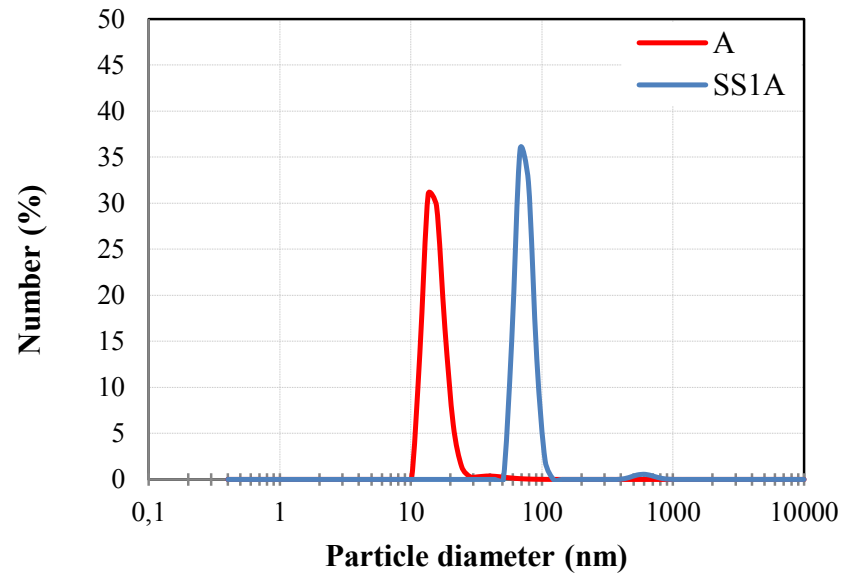

FIGURE 3. Boehmite nanoparticle size distribution for the initial boehmite (A) and the nanostructured material (SS1A).

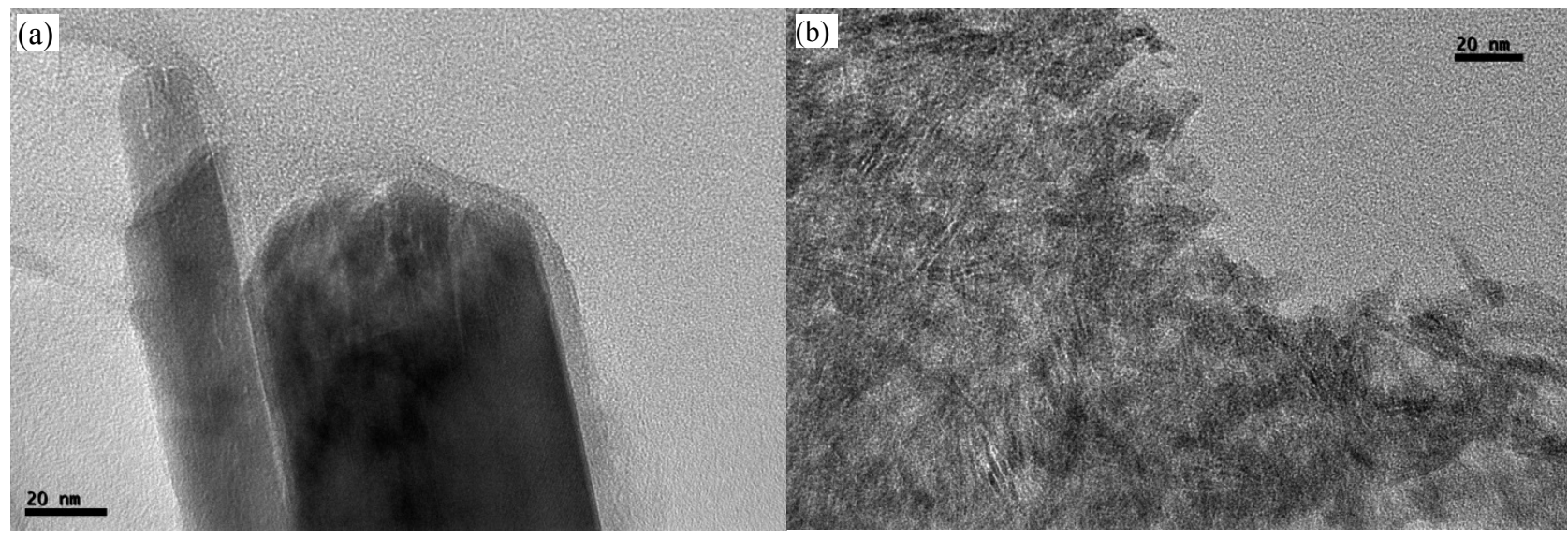

FIGURE 4. TEM images of boehmite nanoparticles. 

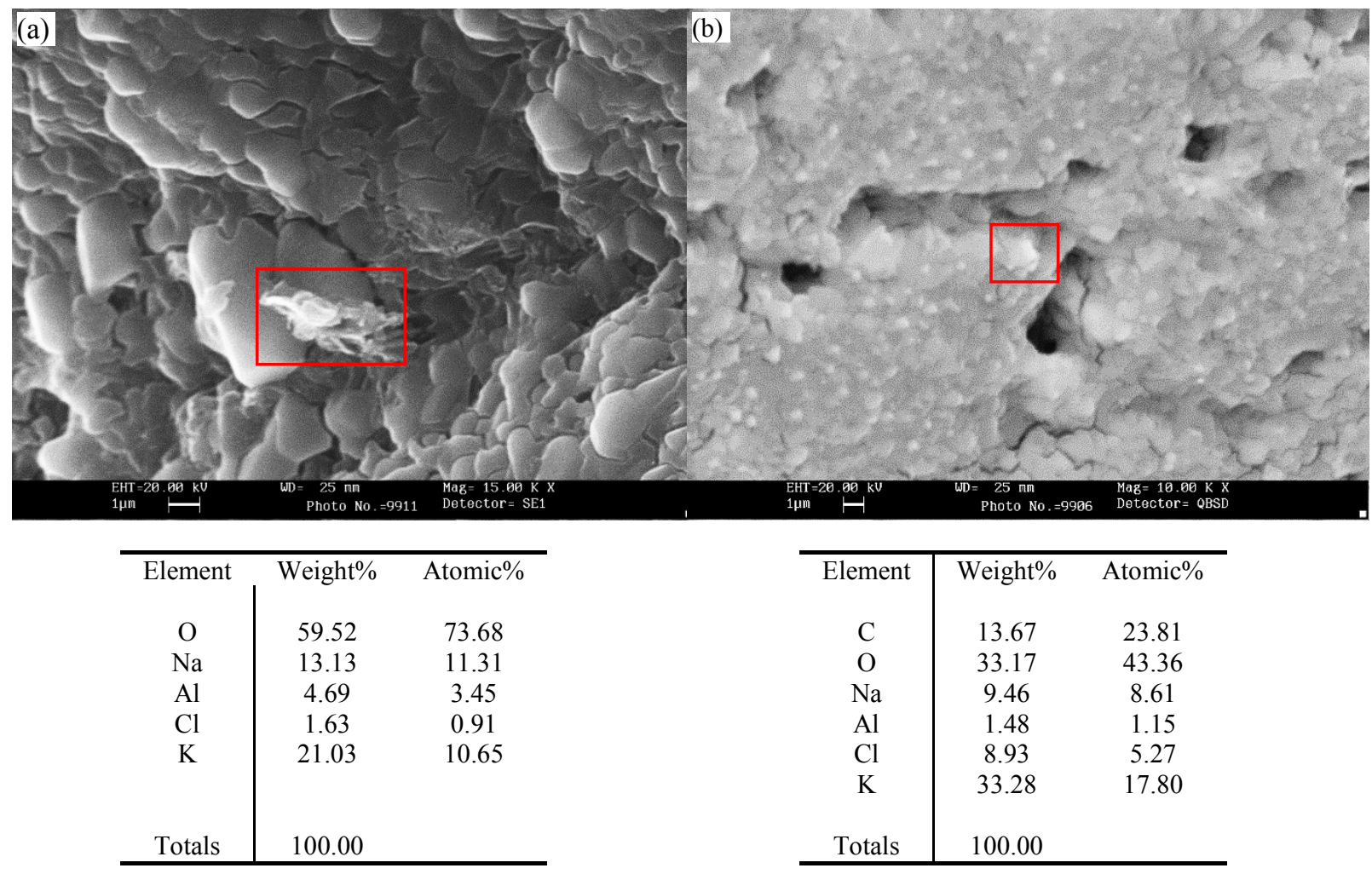

\begin{tabular}{c|cc}
\hline Element & Weight $\%$ & Atomic\% \\
& & \\
$\mathrm{O}$ & 59.52 & 73.68 \\
$\mathrm{Na}$ & 13.13 & 11.31 \\
$\mathrm{Al}$ & 4.69 & 3.45 \\
$\mathrm{Cl}$ & 1.63 & 0.91 \\
$\mathrm{~K}$ & 21.03 & 10.65 \\
& & \\
& & \\
Totals & 100.00 & \\
\hline
\end{tabular}

\begin{tabular}{c|cc}
\hline Element & Weight\% & Atomic\% \\
& & \\
$\mathrm{C}$ & 13.67 & 23.81 \\
$\mathrm{O}$ & 33.17 & 43.36 \\
$\mathrm{Na}$ & 9.46 & 8.61 \\
$\mathrm{Al}$ & 1.48 & 1.15 \\
$\mathrm{Cl}$ & 8.93 & 5.27 \\
$\mathrm{~K}$ & 33.28 & 17.80 \\
& & \\
Totals & 100.00 & \\
\hline
\end{tabular}

FIGURE 5. SEM images of SS1A and their EDX analysis. SE image (a) and QBSD image (b).

\section{Calorimetric Analysis}

Our main objective consists of improving the thermal performance of the nanostructured material. This starts by measuring the $c_{p}$ of the raw SS in a precise manner. These results would allow comparing the value of this thermophysical property in the pure salt with the salt containing nanoparticles. The $c_{p}$ of raw boehmite has been also evaluated in order to establish a theoretical value of the specific heat of the mixture by applying the traditional mixing rule. Results of the specific heat capacity of A, SS and SS1A (theoretical and experimental) are presented in Table 1. In addition, the enhancenments in $\mathrm{c}_{\mathrm{p}}$ obtained with the addition of nanoparticles have been calculated from the experimental SS1A results. The collected data for the SS and A are in concordance with the results provided by the specialized literature $[2,8]$. The results indicate that A has a lower $\mathrm{c}_{\mathrm{p}}$ than the SS and therefore, their mixture may have a decrease of this property with respect to the SS. However, an improvement has been obtained for any of the temperatures, being especially high in the solid state. The observed behavior is in agreement with the findings of other authors [2,3]. Further work is needed to understand this performance and investigate the factors that are affecting this property.

TABLE 1. Specific heat and SD of boehmite (A), solar salt (SS) and final nanofluids (SS1A) at the studied temperatures.

\begin{tabular}{cccc}
\hline \multirow{2}{*}{ Sample } & \multicolumn{3}{c}{ Specific heat, $\mathbf{c}_{\mathbf{p}}\left(\mathbf{J} / \mathbf{g}^{\mathbf{}} \mathbf{C}\right)$} \\
\cline { 2 - 4 } & $\mathbf{1 9 6}^{\mathbf{0}} \mathbf{C}$ & $\mathbf{2 5 6}^{\mathbf{}} \mathbf{C}$ & $\mathbf{3 9 6}^{\circ} \mathbf{C}$ \\
\hline A & $1.195 \pm 0.039$ & $1.237 \pm 0.009$ & $1.471 \pm 0.031$ \\
SS & $1.424 \pm 0.020$ & $1.552 \pm 0.002$ & $1.568 \pm 0.025$ \\
SS1A (Theor.) & 1.421 & 1.548 & 1.567 \\
SS1A (Exp.) & $1.693 \pm 0.025$ & $1.631 \pm 0.027$ & $1.618 \pm 0.028$ \\
Enhanc. (\%) & 18.87 & 5.07 & 3.19 \\
\hline
\end{tabular}




\section{CONCLUSIONS}

A novel nanomaterial composed by boehmite nanoparticles dispersed in a matrix of molten solar salt (an inorganic salt based on nitrates) is proposed as an effective thermal storage material in solar power plants. The initial materials have been characterized prior to the synthesis in order to know their suitability to the final application. Thermal analysis revealed that boehmite undergoes several dehydration events at the working temperature range but they seem to be irreversible. Solar salt remains stable with little weight changes at these temperatures. The crystalline structure was analyzed by XRD and the loss of crystallinity may be related to the thermal dehydration of boehmite. In addition, DLS and TEM were employed to analyze the initial nanoparticle size $(13 \mathrm{~nm})$ and shape (laminar). The synthetized nanomaterial (SS1A) has been also characterized. Its thermal stability results to be very similar to that of the SS with no weight changes. Finally, the nanoparticle size remains in the nanometric range (68 $\mathrm{nm}$ ) after the mixing and manufacturing procedure, which is quite important to obtain improvements of the specific heat. This property was also analyzed and enhancements were found both in the solid and the liquid state compared to the base SS. In addition, the specific heat results of both boehmite and the SS are in the range of the published values.

Further characterization of the nanostructured material is needed in order to understand the mechanism responsible of the enhancement of thermal properties respect to the base salt. Future work includes also the measuring of thermal conductivity, viscosity and cost estimation.

\section{ACKNOWLEDGMENTS}

The authors would like to thank Izaskun Marañón and Gorka Imbuluzqueta for performing the DSC measurements, to Margot Llosa and Alfredo Tanaka for their support for the DLS measurements and results analysis and to the Universitat Jaume I for the SEM and TEM images used in this work. Finally, we show gratitude to the Basque Governement for the economic support given to the Etortek 2014 project on thermal storage and the UPV/EHU for funding the PhD thesis of Belén Muñoz Sánchez through Zabalduz research program.

\section{REFERENCES}

1. D. Shin and D. Banerjee, International Journal of Structural Changes in Solids - Mechanics and Applications, 2 (2), 25-31 (2010).

2. P. Andreu-Cabedo et al., Nanoscale research letters, 9(1):582, (2014).

3. M. X. Ho, C. Pan, International Journal of Heat and Mass Transfer, 70, 174-84 (2014).

4. W. Brostow and T. Datashvili, Chemistry and Chemical Technology, 2(1), 27-32 (2008).

5. T. Tsukada and K. Okadab, Journal of Materials Chemistry, 9, 549-553 (1999).

6. T. Bauer et al., "Sodium nitrate for high temperature latent storage" in 11th International Conference on Thermal Energy Storage-Effstock - 2009, pp. 14-7.

7. A.C. Vieira Coelho et al., Matéria (Rio de Janeiro), 13(2), 329-341 (2008).

8. B. Hemingway and R. Robie, J. A. Apps, American Mineralogist, 76, $445-57$ (1991). 